\title{
PRÁCTICAS PARA LA ENSEÑANZA Y EL APRENDIZAJE DE HABILIDADES DIGITALES EN LA EDUCACIÓN SUPERIOR: una revisión sistemática en la literatura
}

\author{
Fernando José Spanhol' \\ Ana María Martín-Cuadrado² \\ Natana Lopes Pereira ${ }^{3}$
}

\begin{abstract}
RESUMEN
Esta investigación tiene como objetivo identificar prácticas de enseñanza para la enseñanza y el aprendizaje en competencias digitales en la educación superior. Para llevar a cabo este estudio, se realizó una revisión sistemática y se realizaron búsquedas complementarias, basadas en el enfoque cuantitativo, para identificar producciones bibliográficas sobre este tema. Como resultado, es posible mapear la investigación aplicada por año y país, para identificar prácticas y modelos integrados al proceso de construcción del conocimiento, y las tecnologías utilizadas en ellos para mejorar las habilidades digitales de los maestros y (o) estudiantes. Así, se descubrió que las investigaciones seleccionadas se llevaron a cabo principalmente en España entre 2016 y 2018. Además de estos datos, hubo una creciente integración de prácticas colaborativas, a través de entornos virtuales de enseñanza y aprendizaje, para la construcción de conocimiento en habilidades digitales en profesores y alumnos.
\end{abstract}

Palabras clave: Habilidades digitales. Prácticas docentes. Enseñanza superior. Prácticas colaborativas. Entornos virtuales de enseñanza y aprendizaje.

\section{PRÁTICAS PARA ENSINO E APRENDIZAGEM EM COMPETÊNCIAS DIGITAIS NA EDUCAÇÃO SUPERIOR: uma revisão sistemática na literatura}

\section{RESUMO}

Esta pesquisa tem como objetivo identificar práticas didáticas para ensino e aprendizagem em competências digitais no ensino superior. Para realização deste estudo implementou-se uma revisão de forma sistemática, e buscas complementares visando, a partir da abordagem quantitativa, identificar

\footnotetext{
${ }^{1}$ Doutorado em Mídia e Conhecimento. Professor da Universidade Federal de Santa Catarina. Araranguá, Santa Catarina, Brasil. Orcid iD: http://orcid.org/0000-0003-0151-4671. E-mail: fernando.spanhol@ufsc.br

${ }^{2}$ Doctorada en Ciencias de la Educación. Universidad Nacional de Educación a Distancia, UNED. Madrid, España. Orcid iD : http://orcid.org/0000-0001-7579-3701 E-mail: amartin@edu.uned.es

${ }^{3}$ Mestrado em Tecnologias da Informação e Comunicação. Universidade Federal de Santa Catarina, Araranguá, Santa Catarina, Brasil. Orcid iD: http://orcid.org/0000-0002-2942-7011. E-mail:

natanapereiralopes@hotmail.com
} 
produções bibliográficas sobre tal temática. Como resultado pode-se mapear as pesquisas aplicadas por ano e país, identificar práticas e modelos integrados ao processo de construção do conhecimento, e tecnologias utilizadas nos mesmos para aprimoramento das competências digitais dos docentes e (ou) discentes. Assim, constatou-se que as pesquisas selecionadas foram realizadas principalmente na Espanha entre 2016 e 2018. Além de tais dados evidenciou-se a crescente integração de práticas colaborativas, por meio de ambientes virtuais de ensino e aprendizagem, para construção do conhecimento em competências digitais nos docentes e discentes.

Palavras-chave: Competências digitais. Práticas de ensino. Ensino superior. Práticas colaborativas. Ambientes virtuais de ensino e aprendizagem.

\title{
PRACTICES FOR TEACHING AND LEARNING IN DIGITAL SKILLS IN HIGHER EDUCATION: a systematic review of the literature
}

\begin{abstract}
This research aims to identify teaching practices for teaching and learning in digital competences in higher education. To carry out this study, a systematic review and complementary searches were implemented, aiming, from the quantitative approach, to identify bibliographic productions on this theme. As a result, it is possible to map the applied research by year and country, identify practices and models integrated into the process of building knowledge and technologies used in them to improve the digital skills of teachers and (or) students. Thus, it was found that the selected surveys were carried out mainly in Spain between 2016 and 2018. In addition to such data, the growing integration of collaborative practices was evidenced, through virtual teaching and learning environments, for the construction of knowledge on digital competences in teachers and students.
\end{abstract}

Keywords: Digital skills. Teaching practices. University education. Collaborative practices. Virtual teaching and learning environments

\section{INTRODUCCIÓN}

Debido a la adhesión de las Tecnologías de la Información y la Comunicación (TIC) en la educación superior, y debido al uso constante de las tecnologías digitales en las prácticas diarias, el desarrollo y la mejora de nuevas competencias con respecto al uso de los recursos tecnológicos en los campos académico y profesional es actualmente esencial. Kuhn (2017) enfatiza que las TIC están cambiando gradualmente la forma en que se construye el conocimiento en la sociedad, transformando las habilidades 
necesarias para el éxito en las actividades profesionales y para el progreso cognitivo, de acuerdo con las tendencias tecnológicas adherentes.

A partir de este escenario, surgen una serie de estudios sobre habilidades digitales en Europa, ya que se ha convertido en una de las ocho (8) competencias clave para el ciudadano europeo. Son evidentes que el Marco Europeo de Competencia Digital para Ciudadanos - DigComp - y su evolución descrita en el Marco de Competencias Digitales para Educadores - DigCompEdu - se destacan, apuntando a acciones para medir, capturar las competencias digitales que necesitan los docentes (DigCompEdu), y ciudadanos (DigComp), a través de un modelo coherente que permite la evaluación y el desarrollo de habilidades pedagógicas digitales (Redecker, 2017).

En este contexto, también se destaca el programa "Iniciativa Nacional de Habilidades Digitales de Portugal", que tiene como objetivo capacitar y calificar las habilidades digitales de la población portuguesa para el uso efectivo de las TICs. Este programa adapta las acciones de DigComp 2.1 a la realidad del país (Diário de la República, 2019). Además de estas acciones y para comprender el nivel de competencia digital de los brasileños, la investigación realizada por Google tiene como objetivo identificar qué habilidades dominan los ciudadanos a través del Índice de Habilidades Digitales (Digital Skills Index).

Este índice analiza a través de cinco (5) dimensiones, en una escala de cero (0) a cinco (5), las competencias consideradas como nivel básico, como la navegación y el uso de tecnologías, y el conocimiento avanzado, como la programación. El índice promedio de habilidades digitales de los brasileños fue de tres (3), enfatizando así la necesidad de acciones dirigidas a mejorar las habilidades digitales de los ciudadanos (Lima, 2019).

Benali, Kaddouri y Azzima (2018) enfatizan que en los últimos años la competencia digital se ha convertido en un concepto importante en la sociedad del conocimiento. Además, se ha convertido en un elemento clave para la construcción del conocimiento pedagógico, que requiere la práctica y la mejora de las habilidades digitales de profesores y estudiantes. 
Por lo tanto, a partir de este tema evidenciado por Benali, Kaddouri y Azzima (2018) surgen algunas preguntas sobre los modelos didácticos aplicados en las instituciones de educación superior para mejorar, desarrollar las habilidades digitales de maestros y estudiantes.

En este sesgo, esta investigación tiene como objetivo identificar prácticas didácticas para la enseñanza y el aprendizaje en competencias digitales en la educación superior. Con este fin, se realizó una revisión sistemática (artículos) y búsquedas complementarias (tesis), descritas en la sección tres para identificar, mediante análisis cuantitativo, producciones bibliográficas sobre dicho tema. El análisis permitió el mapeo de las producciones seleccionadas por año y país, así como la identificación de prácticas, modelos integrados al proceso de construcción de conocimiento y tecnologías utilizadas para mejorar las competencias digitales de educadores y (o) estudiantes.

\section{FUNDACIÓN TEÓRICA}

La competencia digital se considera un conjunto de conocimientos, habilidades y actitudes necesarias para usar las tecnologías digitales para permitirle realizar tareas variadas, como comunicarse, evaluar información, colaborar, crear y compartir contenido, y construir conocimiento de manera crítica, creativa y autónoma, flexible, ética y reflexiva, para el trabajo el ocio, la participación, el aprendizaje y la socialización (Ferrari, 2012). Se identifica en el marco de referencia europeo como una de las ocho competencias clave ${ }^{4}$ para las personas para su desarrollo personal, interacción interpersonal, ciudadanía, inclusión social y empleo (Parlamento y Consejo Europeo, 2006).

Actualmente se considera la competencia más relevante debido a su transversalidad en relación con todas las demás competencias clave

4 Ocho habilidades: comunicación en la lengua materna; Comunicación en lenguas extranjeras; Competencia matemática y habilidades básicas en ciencia y tecnología; Competencia digital; Aprender a aprender; Competencias sociales y cívicas; Espíritu de iniciativa y espíritu emprendedor; y conciencia cultural y expresión 
(Unesco, 2013; Becker et al., 2017; Vuorikari et al., 2016). Desde este escenario, algunos estudios en Europa tienen como objetivo medir la competencia digital de la población y el impacto de su inclusión en las propuestas curriculares de las instituciones educativas (European Comission, 2017; Vuorikari et al., 2016). Entre varias encuestas, DigComp destaca que tiene como objetivo satisfacer la nueva demanda de ciudadanos digitalmente competentes, donde además de la alfabetización digital, se necesitan algunas habilidades que guíen el conocimiento de los profesionales de las TIC.

De acuerdo con Vuorikari et al. (2016), esta herramienta tiene como objetivo mejorar la competencia digital de los ciudadanos y ayudar en la construcción de políticas públicas, para apoyar la construcción de competencias digitales. Además de este marco y con el objetivo de promover la competencia digital de sus ciudadanos e impulsar la innovación en la educación, se implementó el Marco para el desarrollo de la competencia digital de los educadores en Europa - DigCompEdu (Redecker, 2017). Tal modelo, según Redecker (2017), está destinado a apoyar los esfuerzos en diferentes ámbitos para promover la competencia digital de los educadores, permitiendo un modelo de referencia común de habilidades que necesitan los maestros actuales del siglo XXI (Redecker, 2017).

Por lo tanto, García-Pérez, Santos-Delgado y Buzón-García (2016) enfatizan que uno de los factores clave para la innovación y la mejora educativa es la capacitación docente en TIC, como lo destacan varios informes internacionales, incluida la capacidad de fomentar la interacción a través de prácticas colaborativas que contribuyen al proceso de comunicación digital, autoeficacia y toma de decisiones (García-Pérez; Santos-Delgado y Buzón-García, 2016). En este contexto, se vuelve extremadamente importante hacer un seguimiento e identificar acciones, prácticas didácticas para mejorar las habilidades digitales de los aprendices y los educadores. 


\section{PROCEDIMENTOS METODOLÓGICOS}

Según Severino (2007), el método científico se puede definir como un conjunto de pasos realizados para lograr un objetivo científico previamente definido y delimitado. Por lo tanto, para cumplir con el objetivo de esta investigación que guía dicho estudio, se realizaron búsquedas sistemáticas en la literatura para identificar investigaciones que aborden las prácticas didácticas construidas en la educación superior para desarrollar, mejorar las habilidades digitales de maestros y estudiantes.

La revisión sistemática, según Ferenhof y Fernandes (2016), consiste en la planificación previa del método de investigación en bases de datos científicas, que sintetizan los resultados recopilados de los trabajos de otros autores. Las bases de datos adoptadas para esta investigación fueron Dialnet ${ }^{\circledR}$ y UNED Biblioteca $\AA^{\circledR}$. Dichas bases de datos se utilizaron para la investigación, porque incluyen trabajos considerados relevantes para el tema de la competencia digital.

Inicialmente las búsquedas se realizaron con la siguiente string: ("DIGITAL COMPETENCES" OR "DIGITAL SKILLS") AND ("HIGHER EDUCATION"). Esta construcción devolvió 81 artículos en la base de datos Dialnetß y 1.508 en la base UNED Bibliotecaß. Para delimitar los resultados, se realizaron nuevas búsquedas, incluido un nuevo término: ("DIGITAL COMPETENCES" OR "DIGITAL SKILLS") AND ("HIGHER EDUCATION") AND ("DIDACTIC" OR "PRACTICE OF TEACHING" OR "METHODOLOGY OF TEACHING"). Además de este término, se aplicó como filtro la restricción de período para artículos revisados por pares publicados de 2013 a 2019 en los idiomas inglés, portugués y español.

Decidimos delimitar el año de publicación desde 2013, debido al período en el que se identificaron los primeros registros de DigComp. Esta string devolvió 8 artículos en la base Dialnet® y 92 documentos en la plataforma UNED Library ${ }^{\circledR}$. 
Marco 1. String de búsqueda

\begin{tabular}{|c|c|c|}
\hline BASE DE DATOS & STRING & RESULTADOS \\
\hline Dialnet® & \multirow{2}{*}{$\begin{array}{l}\text { ("DIGITAL COMPETENCES" OR "DIGITAL } \\
\text { SKILLS") AND ("HIGHER EDUCATION") }\end{array}$} & 81 artículos \\
\hline $\begin{array}{c}\text { UNED } \\
\text { Biblioteca }{ }^{\circledR}\end{array}$ & & 1.508 artículos \\
\hline Dialnetß & \multirow{2}{*}{$\begin{array}{l}\text { ("DIGITAL COMPETENCES" OR "DIGITAL } \\
\text { SKILLS") AND("HIGHER EDUCATION") AND } \\
\text { ("DIDACTIC" OR "PRACTICE OF TEACHING" OR } \\
\text { "METHODOLOGY OF TEACHING") }\end{array}$} & 8 artículos \\
\hline$\underline{\underline{\text { UNED }}} \underline{\underline{\text { iotecaß }}}$ & & 92 artículos \\
\hline
\end{tabular}

Fuente: Elaboración propia.

Además de este método de búsqueda, se realizaron búsquedas complementarias para identificar tesis sobre el mismo tema en las bases de datos TESEO $\AA$, Dialnet $\AA$, UNED Bliblioteca $\AA^{\circledR}$ y e-SpacioUNED $\AA$. En las bases de datos Dialnet ${ }^{\circledR}$ y UNED Bliblioteca ${ }^{\circledR}$ se ha aplicado la siguiente string de búsqueda: ("COMPETENCIAS DIGITALES" AND "EDUCACIÓN SUPERIOR" AND ("PRÁCTICA" OR "DIDÁCTICA" OR "METODOLOGÍA DE ENSEÑANZA") devolviendo 10 y 2 tesis respectivamente. En la plataforma TESEO® la combinación de palabras ("COMPETENCIAS DIGITALES") Y ("EDUCACIÓN SUPERIOR") Y ("DIDÁCTICA"), devuelto 21 tesis. Ya en la base e-SpacioUNED® usó solo la string ("COMPETENCIAS DIGITALES"), ya que la aplicación de las otras palabras no arrojó resultados. Esta búsqueda identificó 11 tesis.

Marco 2. String de la búsqueda complementaria

\begin{tabular}{|c|c|c|}
\hline BASE DE DATOS & STRING & RESULTADOS \\
\hline Dialnet® & \multirow{2}{*}{$\begin{array}{l}\text { ("COMPETENCIAS DIGITALES" AND } \\
\text { "EDUCACIÓN SUPERIOR" AND ("PRÁCTICA" } \\
\text { OR"DIDÁCTICA" OR "METODOLOGÍA DE } \\
\text { ENSEÑANZA") }\end{array}$} & 10 tesis \\
\hline $\begin{array}{l}\text { UNED } \\
\text { Biblioteca® }\end{array}$ & & 2 tesis \\
\hline TESEO® & $\begin{array}{l}\text { COMPETENCIAS DIGITALES Y EDUCACIÓN } \\
\text { SUPERIOR Y DIDÁCTICA }\end{array}$ & 21 tesis \\
\hline e-SpacioUNEDß & ("COMPETENCIAS DIGITALES") & 11 tesis \\
\hline
\end{tabular}

Fuente: Elaboración propia. 
Los resultados de búsqueda (sistemática y complementaria) totalizaron 100 artículos y 44 tesis. Se enfatiza que las búsquedas se realizaron el 23 de mayo de 2019, considerándose artículos indexados en las bases de datos seleccionadas hasta la fecha expuesta.

El proceso de análisis y selección de estudios considerados relevantes para esta investigación se realizó en dos etapas. En primera lectura, e identificación de las variables de este estudio en el título, resumen o palabras clave. Luego, los textos se leyeron para identificar prácticas de enseñanza y aprendizaje en habilidades digitales. En estos pasos se aplicaron algunos criterios para la exclusión o inclusión de artículos, como sigue:

Marco 3. Criterios de exclusión e inclusión.

\begin{tabular}{|c|c|}
\hline (E) CRITERIOS DE EXCLUSIÓN & (I) CRITERIOS DE INCLUSIÓN \\
\hline $\begin{array}{l}\text { (E) No contenga strings de búsqueda } \\
\text { en título, resumen y palabras clave. }\end{array}$ & $\begin{array}{l}\text { (I) Contiene strings de búsqueda en } \\
\text { título, resumen y palabras clave }\end{array}$ \\
\hline $\begin{array}{l}\text { (E) No está disponible para acceder al } \\
\text { texto completo en línea y es gratis. }\end{array}$ & (I) Acceso completo a textos en línea. \\
\hline $\begin{array}{c}\text { (E) Contiene strings de búsqueda, pero } \\
\text { el contexto está fuera del tema de } \\
\text { búsqueda }\end{array}$ & $\begin{array}{c}\text { (I) Adherente al tema, incluso sin las } \\
\text { strings de búsqueda }\end{array}$ \\
\hline $\begin{array}{c}\text { (E) No escrito en portugués, inglés y (O) } \\
\text { español }\end{array}$ & $\begin{array}{c}\text { (I) Estar escrito en portugués, inglés y (o) } \\
\text { español }\end{array}$ \\
\hline
\end{tabular}

Fuente: Elaboración propia.

Luego de definir el portafolio bibliográfico de la investigación, se inició el análisis cuantitativo de los mismos, con el objetivo de identificar la proporción y aplicación de trabajos por año y país. Además de estos elementos, la investigación identificó algunas prácticas y tecnologías utilizadas para contribuir a la construcción del conocimiento del maestro y el alumno, como se describe a lo largo de este artículo. 


\section{RESULTADOS}

Como se describió, la búsqueda sistemática y complementaria arrojó un total de 100 artículos y 44 tesis. De estos, 2 artículos y 2 tesis fueron duplicados. Después del primer paso (lectura del título, resumen y palabras clave), se seleccionaron 43 artículos y 29 tesis para su lectura completa. La segunda etapa resultó en la selección de 24 artículos y 17 tesis consideradas relevantes para la investigación, como se describe el año siguiente de esta sección.

Grafica 1. Selección bibliográfica por pasos.

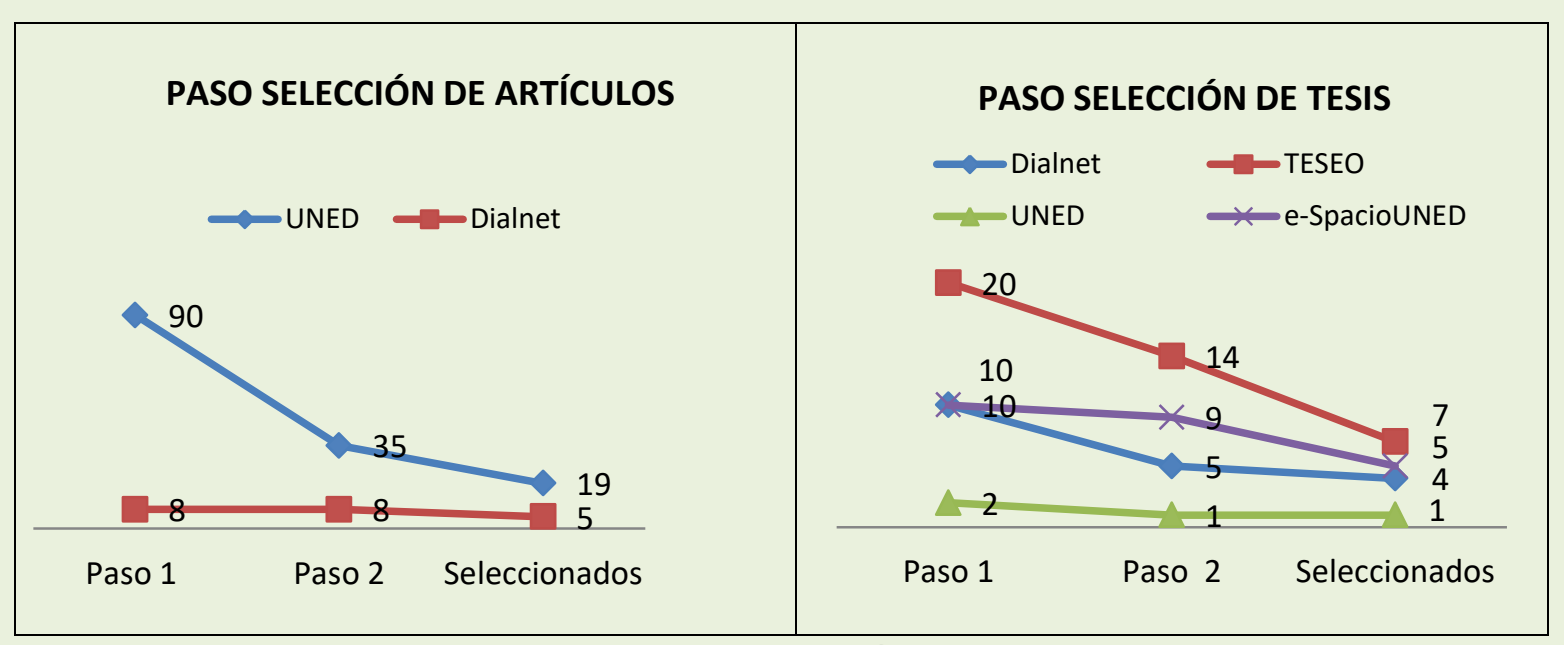

Fuente: Elaboración propia.

Por lo tanto, a partir de la ejecución sistemática de dichas fases de revisión y la aplicación de los criterios de exclusión e inclusión previamente definidos, uno de los resultados de esta investigación se puede obtener de la siguiente cartera bibliográfica de artículos: 
Marco 4. Artículos de cartera bibliográfica: revisión sistemática

\begin{tabular}{|c|c|c|}
\hline TíTULO & AUTOR & AÑO \\
\hline $\begin{array}{c}\text { Which Use Give Teachers at La Laguna } \\
\text { University to ICTs? }\end{array}$ & $\begin{array}{c}\text { Elena, F. V.; Manuel, A. } \\
\text { M. e S., G. G. C }\end{array}$ & 2013 \\
\hline $\begin{array}{l}\text { An interdisciplinary study in initial teacher } \\
\text { training }\end{array}$ & $\begin{array}{l}\text { Rada, V.; Aldecoa, C.; } \\
\text { Cervera, M. e Vidal, C. }\end{array}$ & 2014 \\
\hline $\begin{array}{l}\text { Evaluation of Digital Didactic Skills in Massive } \\
\text { Open Online Courses: a Contribution to the } \\
\text { Latin American Movement }\end{array}$ & $\begin{array}{l}\text { Hernández-Carranza, E. } \\
\text { E.; Romero-Corella, S. I e } \\
\text { Ramírez-Montoya, M. S. }\end{array}$ & 2015 \\
\hline $\begin{array}{l}\text { A Study about Using E-learning Platform } \\
\text { (Moodle) in University Teaching Process }\end{array}$ & Oproiv, G. C. & 2015 \\
\hline $\begin{array}{l}\text { Educating online student teachers to master } \\
\text { professional digital competence: The TPACK- } \\
\text { framework goes online }\end{array}$ & Tømte, C. et al & 2015 \\
\hline $\begin{array}{l}\text { La práctica de la escritura en la lengua } \\
\text { inglesa en una comunidad de } \\
\text { aprendizaje a través de un blog educativo }\end{array}$ & $\begin{array}{c}\text { Arseguet, C. S e Renau, } \\
\text { M. L. }\end{array}$ & 2015 \\
\hline $\begin{array}{c}\text { Educational innovation and digital } \\
\text { competencies: the case of OER in a private } \\
\text { Venezuelan university }\end{array}$ & $\begin{array}{l}\text { Machado, M. S. P. : } \\
\text { Sepðlveda, G. C. T. e } \\
\text { Montoya, M. S. M. }\end{array}$ & 2016 \\
\hline $\begin{array}{c}\text { Virtual empathy as digital competence in } \\
\text { education } 3.0\end{array}$ & $\begin{array}{c}\text { García-Pérez, R.; Santos- } \\
\text { Delgado, J.M. e Buzón- } \\
\text { García, O. }\end{array}$ & 2016 \\
\hline $\begin{array}{c}\text { Teachers' information and communication } \\
\text { technology competences: A structural } \\
\text { approach }\end{array}$ & Almerich, G. et al. 2016 & 2016 \\
\hline $\begin{array}{l}\text { Evaluation and development of digital } \\
\text { competence in future primary school } \\
\text { teachers at the University of Murcia }\end{array}$ & $\begin{array}{c}\text { Porlán, I. G. e Sánchez, J. } \\
\text { L. S. }\end{array}$ & 2016 \\
\hline $\begin{array}{c}\text { Analysis of PLEs' implementation under OER } \\
\text { design as a productive teaching-learning } \\
\text { strategy in Higher Education. A case study at } \\
\text { Universidad Nacional de Educación a } \\
\text { Distancia }\end{array}$ & $\begin{array}{l}\text { Vázquez-Cano, E.; } \\
\text { Martín-Monje, E. e } \\
\text { Castrillo de Larreta- } \\
\text { Azelain, M. D. }\end{array}$ & 2016 \\
\hline $\begin{array}{l}\text { Evaluacion formativa, competencias } \\
\text { comunicativas y TIC en la formacion del } \\
\text { profesorado.(Kaleidoscope)(Ensayo) }\end{array}$ & $\begin{array}{l}\text { Romero-Martin, M. R.; } \\
\text { Castejon-Oliva, F. J.; } \\
\text { Lopez-Pastor, V. M. e } \\
\text { Fraile-Aranda, A. }\end{array}$ & 2017 \\
\hline $\begin{array}{c}\text { Empowering Pre-Service Teachers to Produce } \\
\text { Ubiquitous Flipped Classes }\end{array}$ & $\begin{array}{l}\text { García-Sánchez, S.; } \\
\text { Santos-Espino, J. M. }\end{array}$ & 2017 \\
\hline $\begin{array}{c}\text { Implications of social network sites for } \\
\text { teaching and learning. Where we are and } \\
\text { where we want to go }\end{array}$ & Manca, S. e Ranieri, M. & 2017 \\
\hline $\begin{array}{l}\text { e.Desarrolla el video como objeto de } \\
\text { aprendizaje la competencia digital en la } \\
\text { formacion del profesorado? }\end{array}$ & Garcia Esteban, S. & 2017 \\
\hline
\end{tabular}




\begin{tabular}{|c|c|c|}
\hline $\begin{array}{c}\text { In-service teachers' self-perceptions of digital } \\
\text { competence and OER use as determined by } \\
\text { a xMOOC training course }\end{array}$ & $\begin{array}{c}\text { Ramírez-Montoya, M.; } \\
\text { Mena, J. e Rodríguez- } \\
\text { Arroyo, J. A. }\end{array}$ & 2017 \\
\hline $\begin{array}{c}\text { Professional development in the transition to } \\
\text { online teaching: The voice of entrant online } \\
\text { instructors }\end{array}$ & Adnan, M. & 2017 \\
\hline $\begin{array}{c}\text { Enriching the ICT Competences Of University } \\
\text { Students - A Key Factor For Their Success As } \\
\text { Future Teachers }\end{array}$ & $\begin{array}{c}\text { Gorghiu, G. ; Gorghiu, L. } \\
\text { e Pascale, L. }\end{array}$ & 2018 \\
\hline $\begin{array}{c}\text { Digital competence of Moroccan teachers of } \\
\text { English }\end{array}$ & $\begin{array}{c}\text { Benali, M.; Kaddouri, M. e } \\
\text { Azzimani, T. }\end{array}$ & 2018 \\
\hline $\begin{array}{c}\text { Application of lynda as a teaching resource } \\
\text { in the project of educational innovation in the } \\
\text { excellence of teaching }\end{array}$ & $\begin{array}{c}\text { Rodríguez-Carmona, L. } \\
\text { M. e Herrero, A. P. }\end{array}$ & 2018 \\
\hline $\begin{array}{c}\text { PerLE: An "Open Source", elearning moodle- } \\
\text { based, platform. a study of university } \\
\text { undergraduates' acceptance }\end{array}$ & Servidio, R. e Cronin, M. & 2018 \\
\hline $\begin{array}{c}\text { Experiencias innovadoras de estudiantes } \\
\text { universitarios con software social sobre las } \\
\text { ventajas y debilidades de las tecnologías de } \\
\text { la información y la comunicación en ámbitos } \\
\text { socioeducativos }\end{array}$ & $\begin{array}{c}\text { Galán, J. G; Vázquez- } \\
\text { Cano, e Meneses, E. }\end{array}$ & 2018 \\
\hline $\begin{array}{c}\text { Aprendizaje basado en proyectos para el } \\
\text { desarrollo de la competencia digital docente } \\
\text { en la formación inicial del profesorado }\end{array}$ & & 2018 \\
\hline $\begin{array}{c}\text { Gaming como instrumento educativo para } \\
\text { una educación en } \\
\text { competencias digitales desde los academic } \\
\text { skills centres }\end{array}$ & $\begin{array}{c}\text { García-Quismondo, M. A. } \\
\text { M., e Cruz-Palacios, E. }\end{array}$ & 2018 \\
\hline
\end{tabular}

Fuente: Elaboración propia.

Además de las bibliografías (artículos) seleccionadas en la tabla cuatro (4), la realización de búsquedas complementarias permitió la identificación y selección de 17 tesis ilustradas en la siguiente lista

Marco 5. Portafolio bibliográfico tesis complementaria

\begin{tabular}{|c|c|c|}
\hline TítULO & AUTOR & AÑO \\
\hline $\begin{array}{c}\text { Lecciones aprendidas en programas de } \\
\text { alfabetización informacional en universidades } \\
\text { de iberoamérica. propuesta de buenas } \\
\text { prácticas }\end{array}$ & Uribe Tirado, A. & 2013 \\
\hline $\begin{array}{c}\text { Estudio sobre el uso de internet y sus } \\
\text { aplicaciones en el alumnado de último año de }\end{array}$ & Laguna Segovia, M. I. & 2013 \\
\hline
\end{tabular}




\begin{tabular}{|c|c|c|}
\hline carrera de la universidad de alicante & & \\
\hline $\begin{array}{c}\text { Utilización de wikipedia como recurso docente } \\
\text { en la enseñanza superior }\end{array}$ & Sánchez Martín, L. & 2014 \\
\hline $\begin{array}{l}\text { hipertexto y criticidad: desarrollo de las } \\
\text { competencias digital y medial a través del cine }\end{array}$ & Chávez, M. A. G. R. & 2014 \\
\hline $\begin{array}{l}\text { Las cibercomunidades de aprendizaje y la } \\
\text { formación del profesorado }\end{array}$ & Murua Anzola, I. & 2015 \\
\hline $\begin{array}{l}\text { Evaluación de las Competencias Básicas en TIC } \\
\text { en docentes de educación superior en México }\end{array}$ & Vásquez, M. B. & 2016 \\
\hline $\begin{array}{l}\text { Evaluación de necesidades de formación } \\
\text { continua en competencia digital del } \\
\text { profesorado universitario mexicano para la } \\
\text { sociedad del conocimiento }\end{array}$ & Pérez, K. V.P. & 2016 \\
\hline $\begin{array}{c}\text { Competencias TIC del profesorado de } \\
\text { educación superior y su relación con el uso de } \\
\text { los recursos tecnológicos: análisis de su } \\
\text { formación, uso académico y actitudes desde la } \\
\text { perspectiva de género }\end{array}$ & Díaz, R. P. & 2016 \\
\hline $\begin{array}{c}\text { El desarrollo de la escritura académica con } \\
\text { soporte digital para la formación inicial del } \\
\text { profesorado }\end{array}$ & $\begin{array}{l}\text { Valverde Gonzalez, } \\
\text { M. T. }\end{array}$ & 2016 \\
\hline $\begin{array}{c}\text { El desarrollo de competencias profesionales en } \\
\text { los entornos virtuales de aprendizaje en } \\
\text { ingenierías. el caso de la ingeniería en } \\
\text { informática. }\end{array}$ & López, C. G. & 2016 \\
\hline $\begin{array}{c}\text { Formación de docentes en el uso de las } \\
\text { tecnologías de información y comunicación } \\
\text { para la mejora del proceso enseñanza } \\
\text { aprendizaje en Bolivia }\end{array}$ & Farfán Sossa, S. & 2016 \\
\hline $\begin{array}{c}\text { Educación digital y formación del profesorado } \\
\text { en modalidad semipresencial y virtual (b- } \\
\text { learning y e-learning): Estudios de caso }\end{array}$ & Burgos, J. B. & 2017 \\
\hline $\begin{array}{l}\text { Posibilidades educativas de un mundo virtual 3d, } \\
\text { second life, en el ámbito universitario }\end{array}$ & Nuñez Sanchez, L. & 2017 \\
\hline $\begin{array}{c}\text { Estudio cualitativo sobre el potencial } \\
\text { pedagógico de los entornos digitales móviles } \\
\text { para la construcción de un modelo de } \\
\text { Enseñanza-Aprendizaje en la asignatura de } \\
\text { música en ESO }\end{array}$ & Alises Camacho, M. E. & 2017 \\
\hline $\begin{array}{c}\text { Docentes y escuelas que aprenden en la red. } \\
\text { Estudio sobre la competencia digital, entornos } \\
\text { personales de aprendizaje y entorno } \\
\text { organizacional de aprendizaje de Arizmendi } \\
\text { ikastola }\end{array}$ & Arroyo Sagasta, A. & 2017 \\
\hline
\end{tabular}




\begin{tabular}{|c|c|c|}
\hline $\begin{array}{c}\text { Buenas prácticas TIC: Una experiencia en } \\
\text { educación superior; Best practices ICT: An } \\
\text { experience in higher education }\end{array}$ & Linares Valero, D. & 2018 \\
\hline $\begin{array}{c}\text { Centro de recursos para la enseñanza y el } \\
\text { aprendizaje en una educación basada en } \\
\text { competencias digitales: gaming y videojuegos } \\
\text { para las alfabetizaciones múltiples y la formación } \\
\text { del profesional de la información }\end{array}$ & & \\
\hline
\end{tabular}

Fuente: Elaboración propia.

La encuesta también midió la relación de producciones sobre propuestas didácticas de competencias digitales en educación superior por año. Como se ilustra en el gráfico dos (2), en 2016 y 2017 se concentra un gran número de producciones (artículos y tesis), y en 2018 se identifica un mayor número de artículos publicados sobre el tema.

Grafica 2. Publicaciones por año.

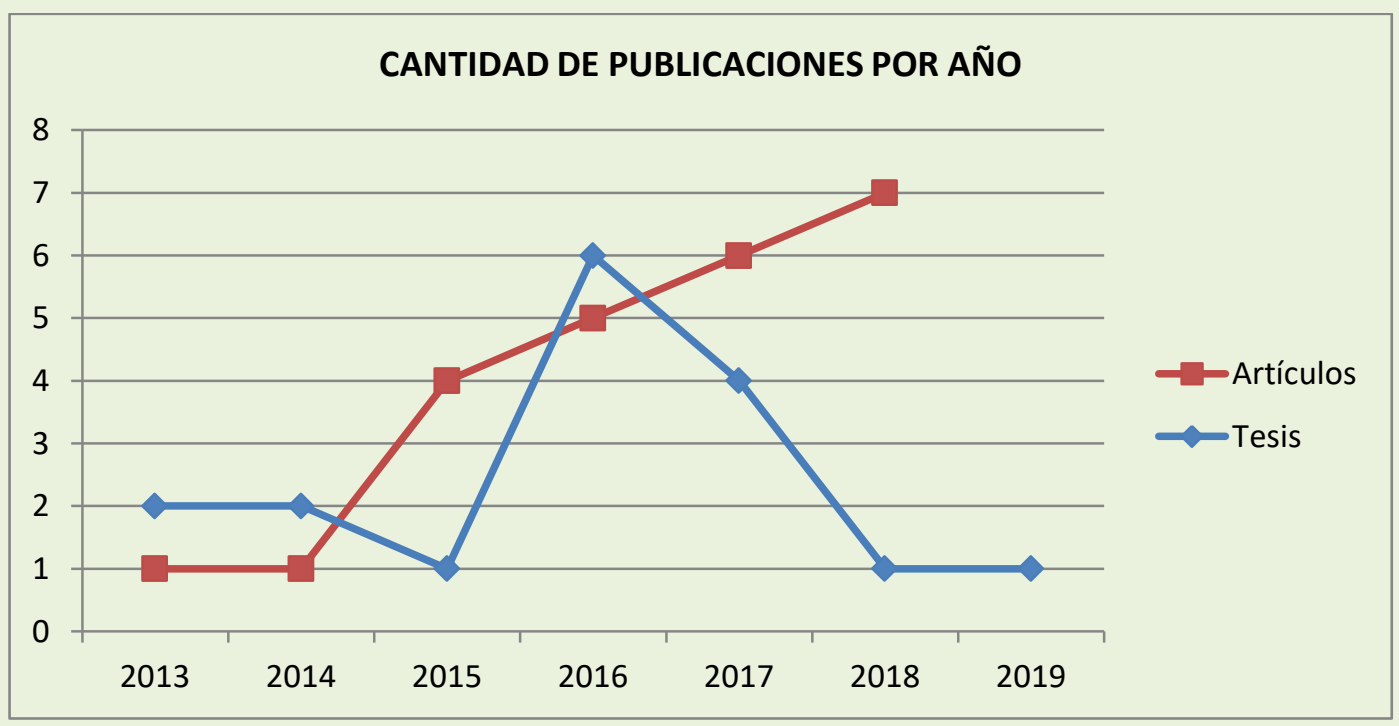

Fuente: Elaboración propia.

En cuanto a la producción por país, hubo una mayor publicación de artículos y tesis en España. Por lo tanto, es de destacar que más del $80 \%$ de las producciones consideradas relevantes para este estudio se realizaron en Europa. Este hecho puede estar relacionado con el área de competencia digital que se considera una de las ocho competencias esenciales para el ciudadano europeo, siendo un objetivo constante de investigación con el objetivo de abordar acciones para su integración en el escenario educativo. 
Grafica 3. Producción bibliográfica por país.

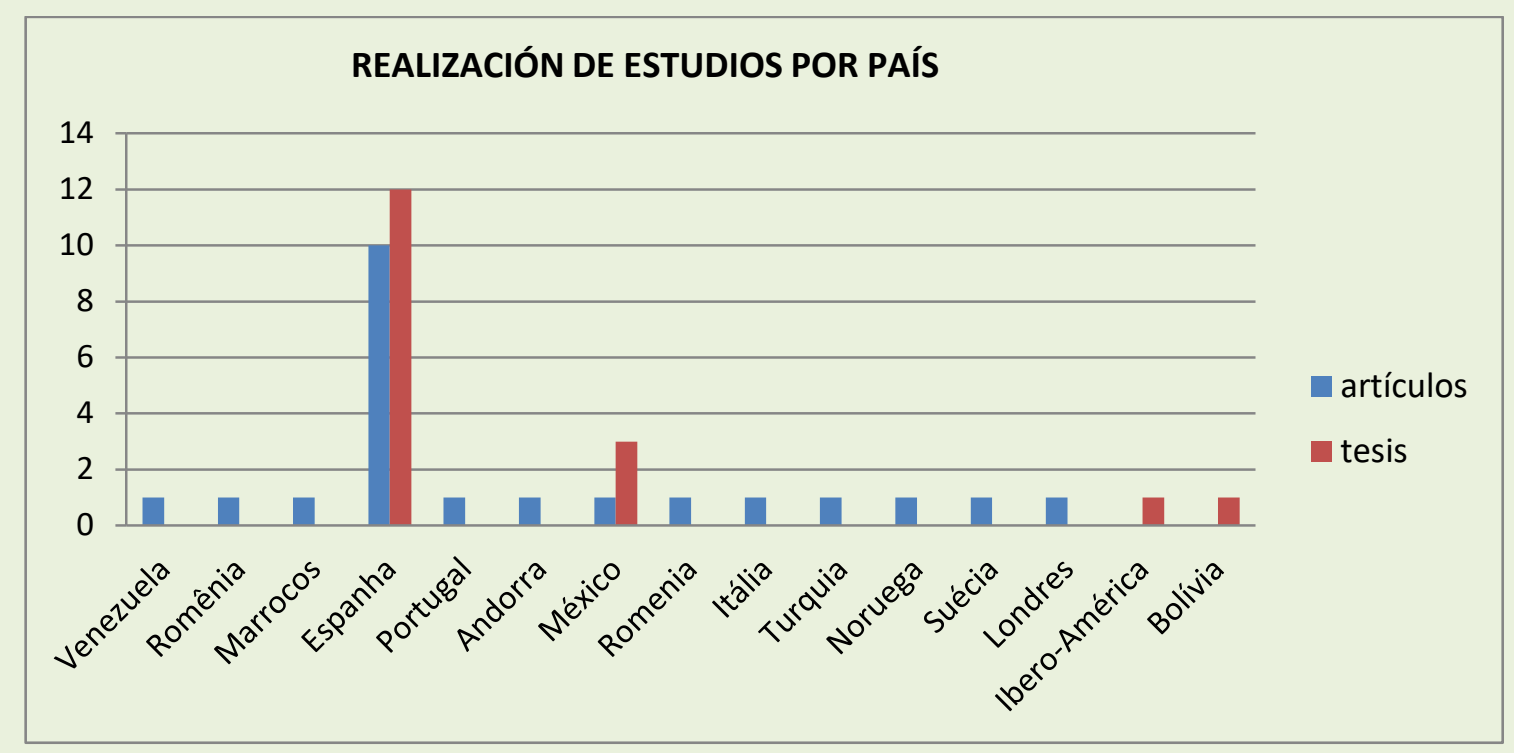

Fuente: Elaboración propia

Además de esta relación, se puede ver que de las producciones relacionadas en la cartera bibliográfica, 13 artículos y 10 tesis abordaron prácticas para la enseñanza y el aprendizaje de la enseñanza, y 11 artículos y 7 tesis para la formación del profesorado.

Grafica 4. Cuantitativo de las prácticas didácticas identificadas para estudiantes o profesores.

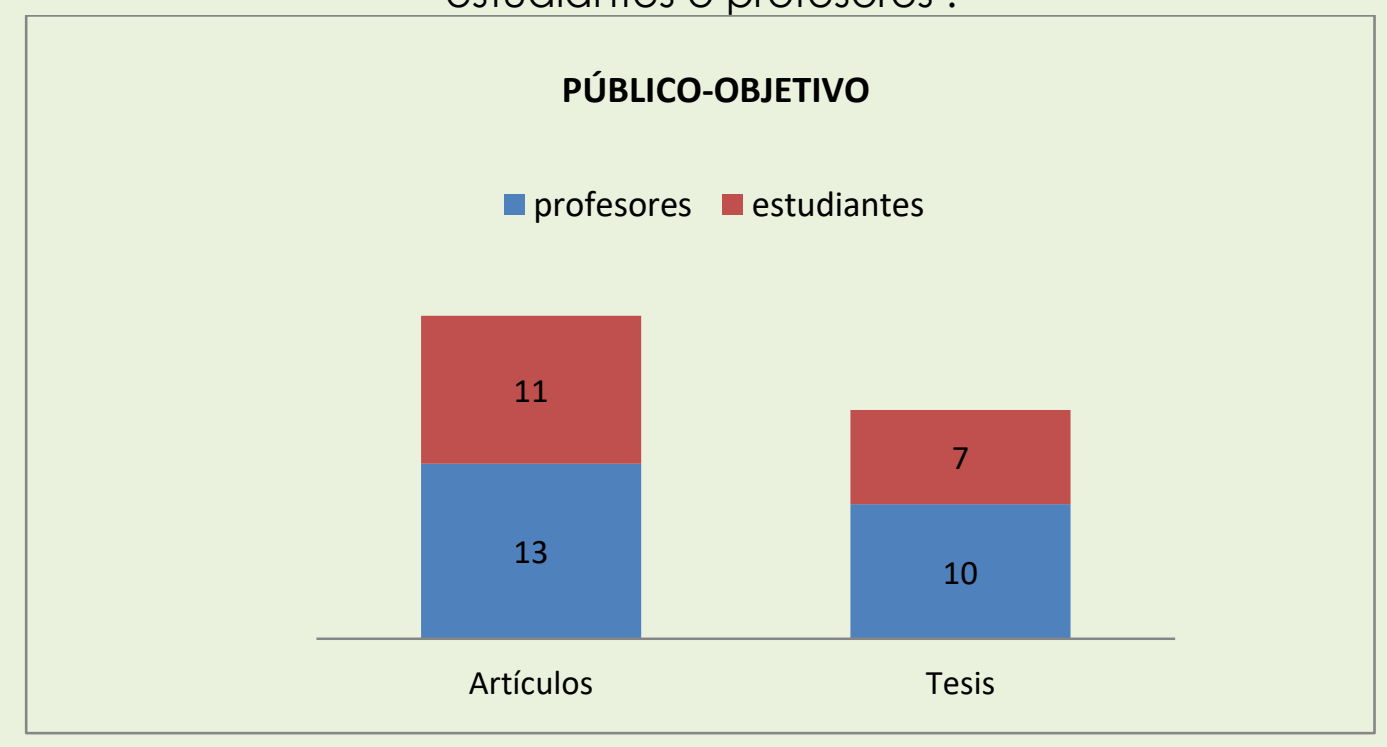

Fuente: Elaboración propia 
A partir de la identificación de algunas prácticas, métodos de enseñanza, se puede resaltar la importancia de las acciones de colaboración. La didáctica abordada en los estudios de Machado, Sepúlveda y Montoya (2016), García-Pérez, Santos-Delgado y Buzón-García (2016), Manca y Ranieri (2017), Elena, Manuel y S. (2013), Servidio y Cronin (2018), Arseguet y Renau (2015), Pérez (2016), Segovia (2013), Murua Anzola (2015) y Sánchez Martín (2014) enfatizan que las propuestas de colaboración a través de un servicio personalizado, comunidades de aprendizaje y redes Las redes sociales, como Facebook, wikis, blogs, entre otras, son esenciales para la construcción del conocimiento en habilidades digitales, ya sea a nivel docente y / o estudiantil.

Además de la importancia de la enseñanza y el aprendizaje de las habilidades digitales a través de procesos de colaboración, se han identificado otros enfoques como el uso de metodologías activas, aulas invertidas, aprendizaje basado en proyectos o (e) problemas centrados en el alumno, y mejor prácticas en la elaboración de objetos de aprendizaje (Rada et al., 2014; García-Sánchez y Santos-Espino, 2017; Rodríguez-Carmona y Presol Herrero, 2018; Alonso-Ferreiro, 2018). Por lo tanto, el potencial de las perspectivas interdisciplinarias para mejorar las habilidades digitales de maestros y estudiantes es evidente (Rada et al., 2014).

Estos métodos enfatizan la importancia de los medios digitales para la elaboración y disponibilidad de objetos de aprendizaje, y abren recursos educativos disponibles en varios dispositivos tecnológicos, especialmente dispositivos móviles (Alises Camacho, 2017; Esteban, 2017; Ramírez-Montoya; Mena y Rodríguez-Arroyo, 2017; García-Quismondo y Cruz-Palacios, 2018; Vázquez-Cano; Martín-Monje y Larreta-Azelain, 2016; Cruz Palacios, 2019; Nuñez Sanchez, 2017; Chávez, 2014). Un ejemplo evidenciado por GarcíaQuismondo y Cruz-Palacios (2018) es el uso de los juegos como un objeto de aprendizaje digital, que proporciona una mayor efectividad para el desarrollo de habilidades digitales.

Dichos objetos digitales y estrategias para su mayor interacción, como la gamificación (García-Quismondo y Cruz-Palacios, 2018; Nuñez Sanchez, 
2017), se aplican en AVEA o Massive Open Online. Cursos - MOOCs - para el desarrollo de habilidades digitales de maestros a distancia y mixtos (Hernández-Carranza; Romero-Corella y Ramírez-Montoya, 2015; López, 2016; Arroyo Sagasta, 2017; Oproiv, 2015; Adnan, 2017). En este sesgo, Tømte et al. (2015) enfatizan la contribución del modelo de aprendizaje mixto, implementado hipermedia educativo sobre las ventajas y desventajas de las TIC en el ámbito socioeducativo y en los servicios sociales a través de redes sociales, como Edublog (Arseguet; Renau, 2015). El uso de tales herramientas permite la implementación de rúbricas de evaluación de habilidades digitales, contribuyendo al monitoreo y evolución de la didáctica en tales habilidades (Galán; Vázquez-Cano; Meneses, 2018).

Una forma importante de entender la combinación de diferentes campos de competencias, uno de ellos es la competencia digital, según Tømte et al. (2015), Burgos (2017) y Benali, Kaddouri y Azzima (2018) es el marco TPACK: conocimiento tecnológico pedagógico del contenido. Tal modelo, de acuerdo con Burgos (2017) tiene como objetivo comprender y describir el conocimiento necesario para que un maestro enseñe y aprenda en un entorno virtual de aprendizaje.

Además de este método, Benali, Kaddouri y Azzima (2018), Porlán y Sánchez (2016) y Farfán Sossa (2016), llevan a cabo estudios adaptando DigCompEdu y DigComp para medir la enseñanza digital y la competencia estudiantil y los Estándares de Competencia Digital UNESCO. Según Benali, Kaddouri y Azzima (2018), la autoevaluación de la competencia digital por parte de docentes y académicos es un enfoque prometedor para fomentar la autorreflexión, la motivación intrínseca para desarrollar habilidades digitales pedagógicas relevantes y el uso innovador de las TIC en las instituciones de educación superior.

Para la innovación con respecto al uso e integración de las TIC en las prácticas de enseñanza y aprendizaje para mejorar las habilidades digitales, Gorghiu, Gorghiu y Pascale (2018) y López (2016) destacan la importancia de desarrollar nuevas herramientas tecnológicas para la construcción del conocimiento de tal manera. En este sesgo, Linares Valero (2018) y Uribe 
(2013) enumeran las mejores prácticas para el uso de las TIC en la educación superior, capaces de desarrollar no solo conocimientos, sino también habilidades digitales en el mundo académico.

De tales estudios se puede destacar que las principales prácticas didácticas seleccionadas en la figura 1 se basan en el modelo mixto de enseñanza y aprendizaje.

Figura 1. Principales prácticas didácticas

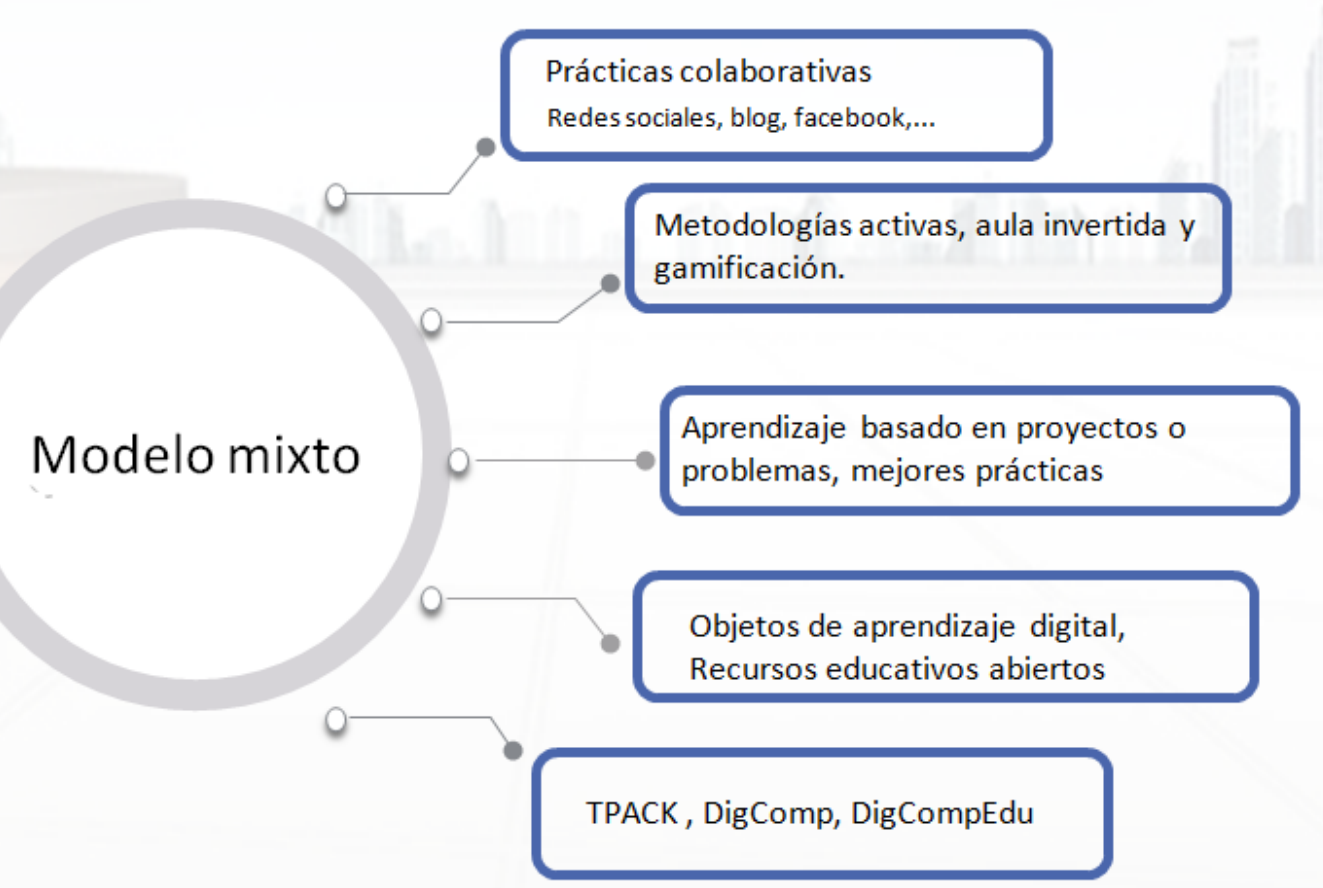

Fuente: Elaboración propia

Por lo tanto, además del método de enseñanza, Burgos (2017) destaca la importancia de integrar tecnologías (enfoque mixto) para mejorar / desarrollar las habilidades digitales de profesores y estudiantes. En este sesgo más allá de las prácticas de enseñanza, podemos destacar las tecnologías más utilizadas, formando una nube de palabras (tecnologías), como se ilustra en la figura dos. 
Figura 2. Nube de palabras. Fuente: wordart.com

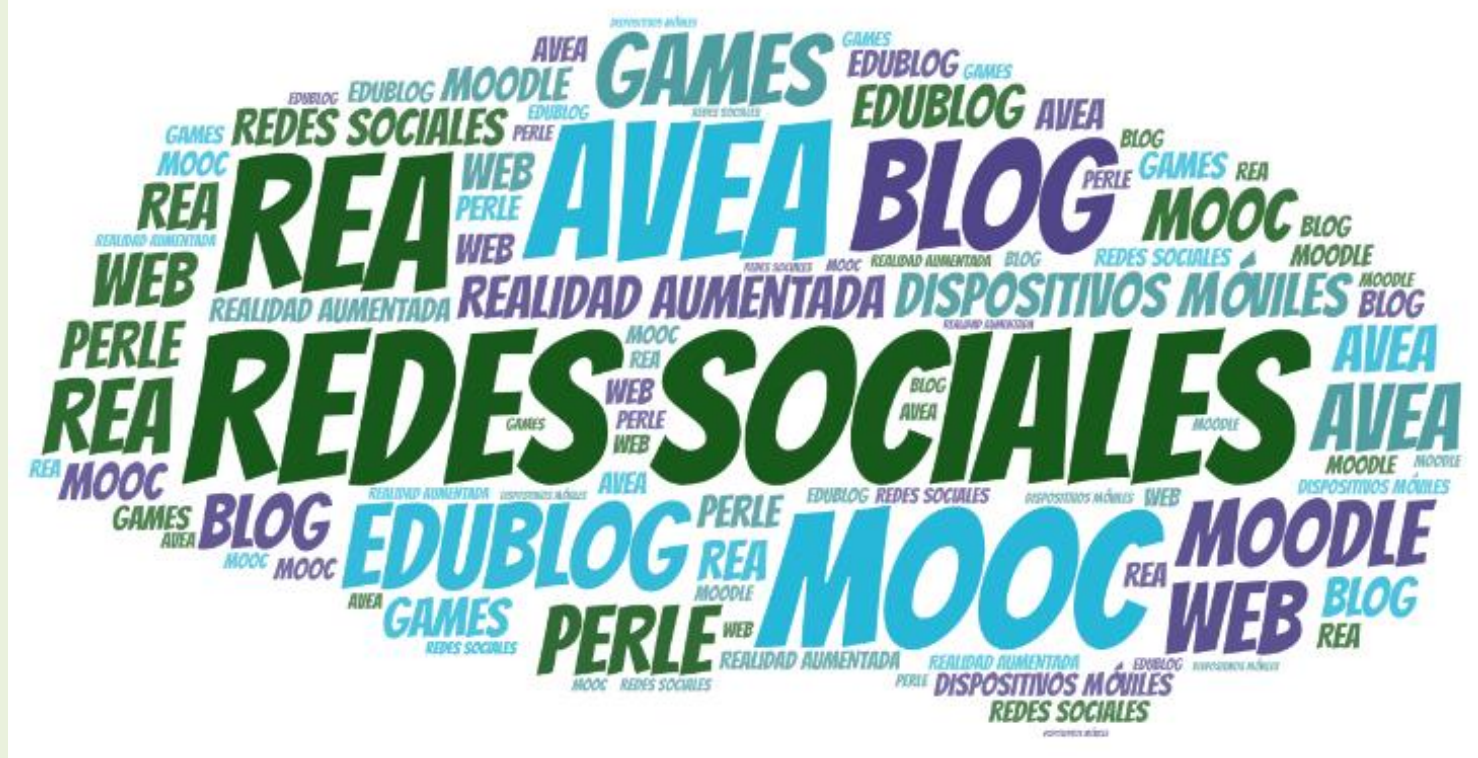

Fuente: wordart.com

\section{CONSIDERACIONES FINALES}

La investigación identificó las prácticas de enseñanza utilizadas por las instituciones de educación superior para mejorar las habilidades digitales de maestros y estudiantes. A partir del análisis de la investigación ilustrada en el portafolio bibliográfico, podemos observar la adhesión al enfoque mixto, principalmente a través de prácticas colaborativas en AVEA, MOOC y redes sociales. Además de estos, otro elemento relevante es la gamificación de tales plataformas, y el uso de objetos virtuales de aprendizaje y recursos educativos abiertos.

Además de las prácticas evidenciadas, la investigación permitió el mapeo de las publicaciones consideradas relevantes para esta investigación, por los criterios de exclusión e inclusión, por año de publicación y país en el que se aplicaron. Por lo tanto, hubo una mayor concentración de estudios en España de 2016 a 2018, y más del $80 \%$ de los estudios se realizaron en Europa.

Otro hecho relevante es la proporcionalidad evidenciada de las prácticas elaboradas para la construcción del conocimiento de los 
estudiantes, relacionados con el tema de competencia digital. Esto identificó la adaptación y aplicación de algunos marcos como DigComp, DigCompEdu, TPACK y el modelo de la UNESCO, para medir y mejorar sus conocimientos tecnológicos y cómo aplicarlos en el proceso de enseñanza y aprendizaje.

Por lo tanto, la investigación resalta la importancia de un enfoque mixto, utilizando metodologías activas y prácticas colaborativas para construir conocimiento en competencias digitales, permitiendo la aplicación de diversas prácticas de enseñanza y aprendizaje. Como continuación de esta investigación, se sugiere llevar a cabo un análisis cualitativo para investigar si tales prácticas contribuyen a mejorar las habilidades digitales en la formación del profesorado para el uso efectivo de las Tecnologías de la Información y la Comunicación en el aula.

\section{REFERENCIAS}

Adnan, M. (2017). Professional development in the transition to online teaching: The voice of entrant online instructors. Recall, [s.l.], v. 30, n. 1, p.88-111. 20 jun. 2017 Cambridge University Press (CUP). http://dx.doi.org/10.1017/s0958344017000106.

Alises Camacho, M. E. (2017). Estudio cualitativo sobre el potencial pedagógico de los entornos digitales móviles para la construcción de un modelo de Enseñanza-Aprendizaje en la asignatura de música en ESO. Universidad Nacional de Educación a Distancia (España). Escuela Internacional de Doctorado. Programa de Doctorado en Comunicación y Educación en Entornos Digitales.

Almerich, G. et al. (2016). Teachers' information and communication technology competences: A structural approach. Computers \& Education, [s.l.], v. 100, p.110-125. Elsevier BV. http://dx.doi.org/10.1016/j.compedu.2016.05.002.

Arroyo Sagasta, A.(2017). Docentes y escuelas que aprenden en la red. Estudio sobre la competencia digital, entornos personales de aprendizaje y entorno organizacional de aprendizaje de Arizmendi ikastola. Universidad Nacional de Educación a Distancia (España). Escuela Internacional de Doctorado. Programa de Doctorado en Comunicación y Educación en Entornos Digitales.

Arseguet, C. S y Renau, M. L. (2015). Practicing your English writing skills in a community learning through an Edublog. EDMETIC, 4, 9-29. 
Becker, S. A. et al. (2017). NMC Horizon Report: 2017 Higher Education Edition. Austin: The New Media Consortium. Recuperado de <http://cdn.nmc.org/media/2017-nmc-horizon-report-he-EN.pdf>. Acesso em: 22 out. 2017.

Benali, M.; Kaddouri, M. y Azzimani, T. (2018). Digital competence of Moroccan teachers of English. International Journal of Education and Development using Information and Communication Technology, Vol.14(2), pp.99-120.

Burgos, J. B. (2017). Educación digital y formación del profesorado en modalidad semipresencial y virtual (b-learning y e-learning). Universidad de Extremadura.

Chávez, M. A. G. R.(2014). Hipertexto y criticidad: desarrollo de las competencias digital y medial a través del cine. Universidad Nacional de Educacion a Distancia (España). Facultad de Educación. Departamento de Didáctica, Organización Escolar y Didácticas Especiales.

Cruz Palacios, E. (2019). Centro de recursos para la enseñanza y el aprendizaje en una educación basada en competencias digitales: gaming y videojuegos para las alfabetizaciones múltiples y la formación del profesional de la información. Universidad Carlos III de Madrid. Departamento de Biblioteconomía y Documentación.

Diário da República. (2019). Presidência Do Conselho De Ministros, Ciência, Tecnologia E Ensino Superior, Educação E Trabalho, Solidariedade E Segurança Social. Despacho n. ${ }^{\circ}$ 1088/201. 2. ${ }^{a}$ série, , N. 22 .p 4184.

Díaz, R. P. (2016).Competencias TIC del profesorado de educación superior y su relación con el uso de los recursos tecnológicos: Competencias TIC del profesorado de educación superior y su relación con el uso de los recursos tecnológicosanálisis de su formación, uso académico y actitudes desde la perspectiva de género Universidad de Salamanca.

Elena, F. V.; Manuel, A. M. y S., G. G. C. (2013). Which Use Give Teachers at La Laguna University to ICTs? Procedia - Social And Behavioral Sciences, [s.l.], v. 93, p.1646-1651. Elsevier BV. http://dx.doi.org/10.1016/j.sbspro.2013.10.095.

European Comission. (2017). Being digitally competent - a task for the 21 st century citizen. Recuperado de https://ec.europa.eu/jrc/en/digcomp. Acesso em: 10 out. 2017.

Farfán Sossa, S. (2016). Formación de docentes en el uso de las tecnologías de información y comunicación para la mejora del proceso enseñanza aprendizaje en Bolivia. Universidad Nacional de Educación a Distancia (España). Facultad de Educación. Departamento de Didáctica, Organización Escolar y Didácticas Especiales. 
Ferenhof, H. A y Fernandes, R. F. (2016). Desmistificando a revisão de literatura como base para redação científica: método SSF. Revista Acb: Biblioteconomia em Santa Catarina, Florianópolis, 21 (3) .550-563.

Ferrari, A. (2012). Digital competence in practice: an analysis of frameworks. Sevilha: JRC-IPTS.

Ferreiro, A. A. (2018). Aprendizaje Basado en Proyectos para el desarrollo de la Competencia Digital Docente en la Formación Inicial del Profesorado. RELATEC: Revista Latinoamericana de Tecnología Educativa, Vol. 17, 924. Recuperado de https://dialnet.unirioja.es/servlet/articulo? codigo=6566732. Acesso em 09 de abr. de 2019.

Galán, J. G; Vázquez-Cano, E., y Meneses, E. L. (2018). Experiencias innovadoras de estudiantes universitarios con software social sobre las ventajas y debilidades de las tecnologías de la información y la comunicación en ámbitos socioeducativos. Hekademos: revista educativa digital, 25, 7-15.

Garcia Esteban, S. (2017). ?Desarrolla el video como objeto de aprendizaje la competencia digital en la formacion del profesorado? Rael Revista Electronica de Linguistica Aplicada, Vol.16, p.85(18).

García-Pérez, R.; Santos-Delgado, J.M. y Buzón-García, O. (2016). Virtual empathy as digital competence in education 3.0. International Journal Of Educational Technology In Higher Education, [s.l.], v. 13, n. 1, p.1-10. Springer Nature. http://dx.doi.org/10.1186/s41239-016-0029-7.

García-Quismondo, M. Á. M., y Cruz-Palacios, E. (2018). Gaming como Instrumento Educativo para una Educación en Competencias Digitales desde los Academic Skills Centres. Revista General de Información y Documentación, [s.I.], v. 28, n. 2, p.489-506. Universidad Complutense de Madrid (UCM). http://dx.doi.org/10.5209/rgid.62836.

García-Sánchez, S. y Santos-Espino, J. M. (2017). Empowering Pre-Service Teachers to Produce Ubiquitous Flipped Classes. Profile Issues In Teachers' Professional Development, [s.l.], v. 19, n. 1, p.169-185. Universidad Nacional de Colombia. http://dx.doi.org/10.15446/profile.v19n1.53857.

Gorghiu, G. ; Gorghiu, y Pascale, L. (2018). ENRICHING THE ICT COMPETENCES OF UNIVERSITY STUDENTS - A KEY FACTOR FOR THEIR SUCCESS AS FUTURE TEACHERS. Journal of Science and Arts, Vol.18(1), pp.183-190.

Hernández-Carranza, E. E.; Romero-Corella, S. I y Ramírez-Montoya, M. S. (2015). Evaluation of Digital Didactic Skills in Massive Open Online Courses: a Contribution to the Latin American Movement. Comunicar, Vol.22(44), pp.81-89.

Kuhn, C. (2017). Are Students Ready to (re)-Design their Personal Learning Environment? The Case of the E-Dynamic. Space. Journal of New 
Approaches in Educational Research, 6(1), 11-19. doi:10.7821/naer.2017.1.185

Laguna Segovia, M. I. (2013). ESTUDIO SOBRE EL USO DE INTERNET Y SUS APLICACIONES EN EL ALUMNADO DE ÚLTIMO AÑO DE CARRERA DE LA UNIVERSIDAD DE ALICANTE. Universidad de Alicante. Didáctica general y didácticas específicas.

LIMA, Dandara. (2019). Digital Skills Index: Google divulga pesquisa pioneira sobre habilidades digitais no Brasil. Recuperado de: <https://www.showmetech.com.br/digital-skills-index-google-divulgapesquisa/>. Acesso em: 06 abr. 2019.

Linares Valero, D. (2018). Buenas prácticas TIC: Una experiencia en educación superior; Best practices ICT: An experience in higher education. Cambio Educativo Para La Justicia Social.

López, C. G. (2016). El Desarrollo De Competencias Profesionales En Los Entornos Virtuales De Aprendizaje En Ingenierías. El Caso De La Ingeniería En Informática. Universidad de Granada. Didáctica de las ciencias experimentales. Doctorado en Enseñanza de las Ciencias y la Tecnología.

Machado, M. S. P.; Sepollveda, G. C. T. y Montoya, M. S. M. (2016). Educational innovation and digital competencies: the case of OER in a private Venezuelan university. International Journal Of Educational Technology In Higher Education, [s.I.], v. 13, n. 1, p.1-10. Springer Nature. http://dx.doi.org/10.1186/s41239-016-0006-1.

Manca, S. y Ranieri, M. (2015). Implications of social network sites for teaching and learning. Where we are and where we want to go. Education And Information Technologies, [s.l.], v. 22, n. 2, p.605-622, 19 ago. 2015. Springer Science and Business Media LLC.http://dx.doi.org/10.1007/s10639-015-9429-x.

Murua Anzola, I. (2015). Las cibercomunidades de aprendizaje y la formación del profesorado. Universidad Nacional de Educacion a Distancia (España). Facultad de Educación. Departamento de Didáctica, Organización Escolar y Didácticas Especiales.

Nuñez Sanchez, L. (2017). Posibilidades Educativas De Un Mundo Virtual 3d, Second Life, En El Ámbito Universitario. Programa Oficial de Doctorado en La Educación en la Sociedad Multicultural. Universidad de Huelva.

Oproiu, G. C. (2015). A Study about Using E-learning Platform (Moodle) in University Teaching Process. Procedia - Social And Behavioral Sciences, [s.l.], v. 180, p.426-432. Elsevier BV. http://dx.doi.org/10.1016/j.sbspro.2015.02.140.

Parlamento y Consejo Europeo. (2006). Recomendación del Parlamento Europeo y del Consejo, de 18 de diciembre de 2006, sobre las competencias clave para el aprendizaje permanente. Diario oficial de 
la Unión Europea, L394/310.

Pérez, K. V. P. (2016). Evaluación de necesidades de formación continua en competencia digital del profesorado universitario mexicano para la sociedad del conocimiento. Universitat Autònoma de Barcelona.

Porlán, I. G. y Sánchez, J. L. S. (2016). Evaluación y desarrollo de la competencia digital de futuros maestros en la Universidad de Murcia. Journal Of New Approaches In Educational Research, [s.I.], v. 6, n. 1, p.51-56.University of Alicante. http://dx.doi.org/10.7821/naer.2016.1.152.

Rada, V.; Aldecoa, C.; Cervera, M. y Vidal, C. (2014). An interdisciplinary study in initial teacher training. Journal of New Approaches in Educational Research, Vol.3, pp.67-74.

Ramírez-Montoya, M.; Mena, J. y Rodríguez-Arroyo, J. A. (2017). In-service teachers' self-perceptions of digital competence and OER use as determined by a XMOOC training course. Computers In Human Behavior, [s.l.], v. 77, p.356-364. Elsevier BV. http://dx.doi.org/10.1016/j.chb.2017.09.010.

Redecker, C. (2017). European Framework for the Digital Competence of Educators: DigCompEdu. Punie, Y. (ed). EUR 28775 EN. Publications Office of the European Union, Luxembourg, ISBN 978-92-79-73494-6, doi:10.2760/159770, JRC1074

Rodríguez-Carmona, L. M. y Herrero, A. P. (2018). La metodología flipped classroom en educación superior. Resultados de uso de LYNDA como recurso para las pre-clases. Revista de Comunicación de La Seeci, [s.l.], n. 46, p.77-91, 16. Sociedad Espanola de Estudios de la Comunicacion Iberoamericana (SEECI). http://dx.doi.org/10.15198/seeci.2018.46.77-92.

Romero-Martin, M. R.; Castejon-Oliva, F. J.; Lopez-Pastor, V. M. y FraileAranda, A. (2017). Evaluacion formativa, competencias comunicativas y TIC en la formacion del profesorado.(Kaleidoscope)(Ensayo). Comunicar , Vol.25 (52), p.73(10).

Sánchez Martín, L. (2014). Utilización De Wikipedia Como Recurso Docente En La Enseñanza Superior. Universidad de Salamanca. Biomateriales: bases estructurales y aplicaciones quirúrgicas.

Servidio, R. y Cronin, M. (2018). PerLE: An "Open Source", ELearning MoodleBased, Platform. A Study of University Undergraduates' Acceptance. Behavioral Sciences, [s.l.], v. 8, n. 7, p.63-70. MDPI AG. http://dx.doi.org/10.3390/bs8070063.

Severino, Antônio Joaquim. (2007). Metodologia do Trabalho Científico. 23. ed. São Paulo: Cortez. p. 304.

Tømte, C. et al. (2015). Educating online student teachers to master professional digital competence: The TPACK-framework goes online. Computers \& Education, [s.I.], v. 84, p.26-35. Elsevier BV. 
http://dx.doi.org/10.1016/j.compedu.2015.01.005.

Unesco. (2013). Global Media and Information Literacy Assessment Framework: Country Readiness and Competencies. Paris.

Uribe Tirado, A. (2013). Lecciones Aprendidas En Programas De Alfabetización Informacional En Universidades De Iberoamérica. Propuesta De Buenas Prácticas. Universidad de Granada. Biblioteconomía y documentación.

Valverde Gonzalez, M. T. (2016). El Desarrollo De La Escritura Académica Con Soporte Digital Para La Formación Inicial Del Profesorado. Universidad de Murcia. Escuela Internacional de Doctorado de la Universidad de Murcia.

Vásquez, M. B. (2016). Evaluación de las Competencias Básicas en TIC en docentes de educación superior en México. UNED. Universidad Nacional de Educación a Distancia (España).

Vázquez-Cano, E.; Martín-Monje, E. y Castrillo de Larreta-Azelain, M. D. (2016). Analysis of PLEs' implementation under OER design as a productive teaching-learning strategy in Higher Education. A case study at Universidad Nacional de Educación a Distancia. Digital Education Review, 29, 62-85.

Vuorikari, R.; Punie, Y.; Carretero Gomez S. y Van Den Brande, G. (2016). DigComp 2.0: The Digital Competence Framework for Citizens. Update Phase 1: The Conceptual Reference Model. Luxembourg Publication Office of the European Union.

Recebido em: 27 de fevereiro de 2020 Aprovado em: 11 de junho de 2020 Publicado em: 30 de julho de 2020

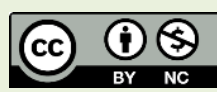

